Classification

Physics Abstracts

$61.25 \mathrm{H}-68.45 \mathrm{G}-6475$

\title{
Polymer adsorption in a poor solvent
}

\author{
A Johner and J. F. Joanny \\ Institut Charles Sadron, 6 rue Boussingault, 67083 Strasbourg Cedex, France
}

(Reçu le 20 septembre 1990, accepté le 31 octobre 1990)

\begin{abstract}
Résumé. - Nous étudions l'adsorption de polymères en mauvais solvant Dans le cas d'une chaîne isolée au contact d'une paroı attractıve nous obtenons une transitıon de mouıllage lorsque la longueur de corrélation thermıque $\xi$ est du même ordre de grandeur que la longueur d'extrapolation $D$ qui caractérise l'adsorption $S_{1} D$ est plus grand que $\xi$, cas de l'adsorption faıble, la chaîne polymère moulle partıellement la surface comme le ferait une gouttelette de liquide, lorsque $D$ est plus petit que $\xi$, cas d'adsorption forte, la chaîne forme une galette d'épaisseur $D$ L'adsorption d'une solution de polymère en mauvais solvant est envisagée unıquement lorsque le polymère moulle la paroı Aux très farbles concentrations volumiques les chaînes adsorbées, constıtuent une solution dıluée à deux dımensıons, le polymère est alors en mauvars ou en bon solvant survant la valeur de l'interaction attractıve entre monomères Lorsque la solution à deux dımensıons est en mauvaıs solvant elle présente une transitıon de démıxtıon du premier ordre analogue à la transition de prémoullage A des concentrations plus élevées, la couche adsorbée devient contınue, son épaisseur augmente et présente une divergence loganthmique quand la concentration volumique atteint la coexistence. Nous étudions également la transition de mouillage de la solution de polymères Les résultats peuvent être appliqués à l'adsorption de copolymères biséquencés en solvant sélectıf Lả transitıon de prémoullage peut également exıster sı la masse molécularre des séquences solubles n'est pas trop élevée
\end{abstract}

\footnotetext{
Abstract. - We discuss polymer adsorption in a poor solvent. For a single chain we find a wetting transition on an attractive wall when the thermal correlation length $\xi$ is of the order of the adsorption length $D$ If $D$ is larger than $\xi$, for weak adsorption, the polymer chain partrally wets the solid surface in the same way as a small liquid drop ; if $D$ is smaller than $\xi$, strong adsorption, the chain forms a flat pancake of thickness $D$ The adsorption of a polymer solution in a poor solvent is considered only when the chains wet the surface At very low bulk concentration the adsorbed chains form a two dimensional dilute polymer solution which is either in a poor solvent or in a good solvent depending on the value of the attractive excluded volume. When this two dimensional solution is in a poor solvent it undergoes a first order demixing transition analogous to the so-called prewetting transition At a higher bulk concentration the surface layer becomes contınuous, its thickness grows and diverges when the bulk concentration reaches the coexistence curve We also study the wetting transition of the polymer solution These results can be applied to diblock copolymer adsorption in a selective solvent The prewetting transition can also exist in this case if the soluble block is not too large
} 


\section{Introduction.}

In our recent work $[1,2]$ we have studied the interfacial behavior of diblock copolymers in the so-called highly selective solvent limit One of the blocks A is in a very poor solvent and tends to form molten regions where the solvent does not penetrate and the other block B is in a good solvent and has a swollen configuration In contact with a wall that attracts the insoluble A sequence and repels the soluble $B$ sequence the copolymer forms a double layer structure with a molten A film in contact with the surface and a B brush dangling into the solvent

In many experimental situations [3], the -solvent does not have such a high selectivity and although the A monomers are in a poor solvent they form dense regions where the solvent can nevertheless penetrate In order to estimate the importance of this effect on the adsorption properties of copolymers we are lead to study first the adsorption of a homopolymer (corresponding to the A block) in a poor solvent

In a poor solvent, the excluded volume parameter $v$ is negative and due to the very low value of the entropy of mixing of the polymer, even at a temperature close to the $\theta$ compensation temperature where $v$ vanishes, the solution phase separates into a polymer dense phase and a polymer dilute phase $[4,5]$. If the molecular weight of the polymer is large the monomer concentration in the dilute phase is extremely low, this makes difficult any experimental study of the properties of the polymer chains in this phase [6]. One expects a very dilute solution of collapsed chains with a finite internal concentration The local structure of the chains is then the same as the local structure of the chans in the dense polymer phase in equilibrium. A collapsed polymer chain should thus be viewed as a small volume of the equilibrium dense phase, at the same temperature [7], the only difference due to the finite size of the chain is the existence of the interface between the polymer globule and the solvent. This suggests that the dominant contribution to the free energy of a collapsed chain is an interfacial free energy quite similarly to the free energy of a droplet of an immiscible liquid in the solvent.

When attracted to a solid surface a collapsed polymer chain takes a configuration that minımizes its free energy The dominant contribution to the free energy being an interfacial contribution, the configuration of the chain is very similar to' that of a small liquid drop of an immiscible liquid wettıng a solid surface ; it forms a small spherical cap characterized by its contact angle If the attractive interaction between the monomers and the interface is small enough the contact angle has a finite value corresponding to a partial wetting situation, for higher values of the attraction, the contact angle vanushes and the chain forms a very flat pancake on the interface, this corresponds to a complete wetting situation One should therefore observe a wetting transition [8] between these two regimes by varying the strength of the attraction or the quality of the solvent (by changing temperature) This wetting behavior of an isolated polymer chain in a poor solvent is discussed in details in section 3 and is interpreted in terms of blobs

If we now consider not a single chain but a solution at a finite but very small concentration, a dilute solution of adsorbed chains is formed on the surface In the strong' adsorption limit where the chains form flat pancakes the adsorbed layer has the structure of a two dimensional dilute polymer solution. The $\theta$ point of a given polymer is lower in two dimensions (for an adsorbed chain) than in three dimensions (in the bulk), eventhough the bulk solution is in a poor solvent, the adsorbed layer can be -either in a good solvent (repulsions between pancakes) if the attraction by the surface is very strong or in a poor solvent (attractions between pancakes) if the attractions by the surface are weaker

As the bulk concentration is increased, the adsorbed layer first becomes continuous and then grows in thickness- The transition between a discontinuous and a continuous layer is smooth if the surface layer is in a good solvent but it is discontinuous if the surface layer is in a 
poor solvent This surface transition is the so-called prewetting transition in the language used for liquid mixtures [6] As for usual wettıng transitions, the thickness of the wettıng layer diverges when the bulk concentration reaches the demixing equilibrium concentration. The transition between a discontinuous and a continuous adsorbed layer and the growth of the layer thickness are described in section 4

The last section gives a brief summary and some possible applications of these results to diblock copolymers

\section{Polymer solution in a poor solvent.}

We first recall the principal results on the Flory theory, of phase separation of a polymer solution in a poor solvent and on the structure of collapsed chains

2.1 Phase Separation in'a POOR solvent. - The free energy per unit volume of a polymer solution in a poor solvent is given in a Flory-Huggins theory by

$$
\frac{F}{k T}=\frac{\phi}{N} \log \phi a^{3}+\frac{v}{2} \phi^{2}+\frac{w^{2}}{6} \phi^{3}
$$

where $\phi$ is the polymer volume fraction, $a$ the size of a monomer and $N$ the degree of polymerization. The first term represents the translational entropy of the chains and the interaction terms are treated as a virial expansion, $v$ is the excluded volume (second virial coefficient), it vanishes at the $\theta$ temperature and is negative in a poor solvent (at a temperature lower than $\theta$ ) and $w^{2}$ is the third virial coefficient of order $a^{6}$

The Flory free energy predicts a phase separation between polymer and solvent at a low temperature with a critical point located at

$$
v_{c}=-\frac{2 w}{N^{1 / 2}}, \quad \phi_{c}=\frac{1}{N^{1 / 2} w}
$$

Below the consolute point the solution separates into a polymer rich and a solvent rich phase. The compositions of these phases are obtained by equating their chemical potential $\mu=\frac{\partial F}{\partial \phi}$ and their osmotic pressure $\pi=\phi \mu-F$. At a temperature lower than the critical point, the concentration in the dilute polymer phase is extremely small and the osmotic pressure vanishes, this gives the concentration in the dense phase and the monomer chemical potential at coexistence

$$
\phi_{\mathrm{d}}=-\frac{3 v}{2 w^{2}}, \quad \frac{\mu_{\mathrm{eq}}}{k T}=-\frac{3 v^{2}}{8 w^{2}}
$$

The Flory free energy does not take into account the fact that in the dilute phase the monomers are localized into the globules formed by the polymer chains it cannot thus be used directly to describe the dilute phase In the next sèction we describe the structure of the dilute phase as a gas of collapsed chains ; for many purposes, it is enough to approximate it as a pure solvent $(\phi=0)$.

In order to study solutions where the concentration is not homogeneous, one should add to the Flory-Huggins free energy (1) terms that take into account the local vanations of concentration. 'This is usually done in a Landau-Ginzburg way by expanding $F^{\prime}$ in powers of the concentration gradient [9]

$$
\frac{F}{k T}=\frac{\phi}{N} \log \phi a^{3}+\frac{v}{2} \phi^{2}+\frac{w^{2}}{6} \phi^{3}+\frac{a^{2}}{24} \frac{(\nabla \phi)^{2}}{\phi}
$$


Contrary to binary mixtures of simple liquids, the dominant contribution to the gradient term is not due to the finite range of the potential but to the elasticity of the polymer chain ; this explains the concentration dependence.

This free energy allows for the calculation of the concentration correlation length $\xi$ and of the interfacial tension $\gamma$ between the two phases at equilibrium at low temperature. Neglecting the entropy term one gets

$$
\xi=-\frac{a w}{3 v}, \quad \frac{\gamma}{k T}=\frac{3 v^{2} a}{16 w^{3}} .
$$

Equations (3)-(5) find a simple interpretation in terms of blobs In the concentrated polymer phase at length scales smaller than $\xi$, the attractive interactions are small and the chains are Gaussian It is then useful to group the monomers into thermal blobs of size $\xi$; each blob contains $g \sim \xi^{2}$ monomers and the concentration inside the blobs $g / \xi^{3}$ is of the same order as the coexistence concentration given by (3) because $w \sim a^{3}$. The attractive excluded volume interaction between the blobs is of order $k T$, the dense polymer phase should thus be considered as a closepacking of thermal blobs. Finally, the interfacial tension $\gamma$ corresponds to an energy $k T$ per blob on the interface and scales as $k T / \xi^{2}$ in agreement with (5)

In this section we have only discussed a poor solvent solution not too close to the consolute point such that the correlation length $\xi$ is smaller than the Gaussian radius of the chains $R=N^{1 / 2} a\left(-v N^{1 / 2}>1\right)$, throughout the remaining of the paper only this limit is considered

2.2 COLLAPSED CHAINS - The structure of a collapsed chain with an internal monomer volume fraction $\phi$ can be studied in a similar way to that of the dense coexistence phase. The free energy per unit volume $F$ of a collapsed chain in a poor solvent is given by equation (4) without the entropy term because we only consider monomers of the same chain and there is no mixing entropy,

$$
\frac{F}{k T}=\frac{v}{2} \phi^{2}+\frac{w^{2}}{6} \phi^{3}+\frac{a^{2}}{24} \frac{(\nabla \phi)^{2}}{\phi} .
$$

As earlier the two first terms describe the interactions between monomers, the entropic elasticity of the polymer being described by the last term [5].

The monomer concentration inside the chain is obtained by minimizing the total free energy at a constant number of monomers $N$ This constraint can be taken into account by introducing a Lagrange multıpher $\lambda$ and minımızıng the free energy functional $\mathcal{F}=\int \mathrm{d} r(F-\lambda \phi)$ with respect to $\phi$.

As shown by Moore [10], the concentration is constant over most of the chain volume except in an interfacial layer of size $\xi$ given by (5) much smaller than the radius $R$ The important point is then that the Lagrange multiplier $\lambda$ must be to a very good approxımation equal to the chemical potential at coexistence $\mu_{\text {eq }}$ given by (3). This is due to the fact that the chemical potential of the solvent is constant inside and outside the chain where we have a pure solvent, and therefore that the osmotic pressure inside the chain vanishes. This is precisely the condition leading to equation (3).

The concentration inside a collapsed chain is thus exactly that of the dense phase on the phase diagram at the same temperature given by (3). The structure of a collapsed chain can then also be described as a closepacked assembly of Gaussian blobs of size $\xi$. The radius of the chain is

$$
R=\left(3 N / 4 \pi \phi_{\mathrm{d}}\right)^{1 / 3} \sim N^{1 / 3}\left(\frac{w^{2}}{-v}\right)^{1 / 3}
$$


If we now take as the zero of the chemical potential the chemical potential at coexistence $\mu_{\text {eq }}$, the monomers inside a collapsed chain have a chemical potential equal to zero. The free energy of a collapsed polymer chain is only due to the monomers in the interfacial layer and is governed by the interfacial tension $\gamma$. We may thus write the chemical potential of a collapsed chain as

$$
\mu_{\mathrm{ch}}=\log \phi a^{3} / N+4 \pi R^{2} \gamma .
$$

The concentration on the dilute branch of the phase diagram at low temperature is then obtained by equating the chain chemical potential to zero; it decays with molecular weight as $\exp -N^{2 / 3}$ and is thus extremely small as announced.

\section{Single chain adsorption in a poor solvent.}

We now discuss the adsorption of an isolated polymer chain in a poor solvent. Since the chain is locally Gaussian, we start by a brief summary of the adsorption of Gaussian chains and then discuss the adsorption in a poor solvent.

3.1 ADSORPTION OF A GAUSSIAN CHAIN. - The propagator of a Gaussian chain is the solution of a Schrödinger-like equation first proposed by Edwards [11] For a chain adsorbed on a wall, a ground state dominance approximation leads to the following equation for the order parameter $\psi$ related to the local concentration $\phi$ by $\psi^{2}=\phi[12]$

$$
\varepsilon \psi=-\frac{a^{2}}{6} \frac{\partial^{2} \psi}{\partial z^{2}}+\frac{V(z)}{k T} \psi
$$

where $z$ is the distance from the adsorbing wall and $\varepsilon$ is the lowest eigenvalue of the Schrodinger equation, it can be interpreted physically as a chemical potential $V(z)$ is the attractive potential due to the wall that we consider to be short range.

This equation can also be obtained by minimization of the free energy of the chain with a free energy density given by equation $\left(4^{\prime}\right)$ where only the gradient term should be considered, both $v$ and $w$ vanish for a Gaussian chain $\varepsilon$ must then be introduced as a Lagrange multiplier associated to the constant number of monomers in the chain and the external potential $V$ must be added.

We are not interested here in detalls of the concentration profile very close to the wall, it is then sufficient to neglect the attractive potential and replace it by a boundary condition on the logarithmic derivative of $\psi$

$$
\frac{1}{\psi} \frac{\partial \psi}{\partial z}=-\frac{1}{D}
$$

where $D$ is an adsorption length When the potential is mimicked by a localized attraction $V(z)=-k T \delta \delta(z), D$ is inversely proportional to the attraction energy $D=\frac{a^{2}}{6 \delta}$.

The profile of the order parameter decays exponentially from the wall with a decay length $D$ so that the concentration $\phi$ decays exponentially over a length $D / 2$ The eigenvalue $\varepsilon$ is equal to $\varepsilon=-\frac{a^{2}}{6 D^{2}}$. An adsorbed Gaussian chain on a wall forms thus a flat pancake of thickness $D$ independent of molecular weight.

3.2 ADSORPTION IN A POOR SOLVENT. - The same approach can be followed in a poor solvent. One must then add to the concentration gradient term in the free energy the 
interaction terms of equation (4') that provoke the chain collapse. The equation for the order parameter becomes

$$
\varepsilon \psi=-\frac{a^{2}}{6} \frac{\partial^{2} \psi}{\partial z^{2}}+v \psi^{3}+\frac{w^{2}}{2} \psi^{5}
$$

with a boundary condition still given by (9).

It is useful to define dimensionless variables by $\varepsilon=a^{2} / 6 D^{2} \eta, z=D x$ and $\psi=\psi_{\mathrm{d}} f$ where $\psi_{\mathrm{d}}$ is the order parameter for a collapsed chain in the bulk $\psi_{\mathrm{d}}^{2}=\phi_{\mathrm{d}}$. In these units equation (10) 'becomes

$$
\eta f=-\frac{\partial^{2} f}{\partial x^{2}}+\left(\frac{D}{\xi}\right)^{2}\left(-f^{3}+\frac{3 f^{5}}{4}\right)
$$

The concentration profile of an adsorbed chain in a poor solvent depends essentially on the dimensionless parameter $D / \xi$ If $D$ is much smaller than $\xi$ (for-strong adsorption), the interaction terms are negligible and as for a Gaussian chain the polymer forms a flat pancake of thickness $D, \eta$ is then equal to - 1 If $D$ is much larger than $\xi$ (for weaker adsorption), the dominant terms are the interaction terms and $\varepsilon$ is approximately equal to the chemical potential at coexistence $\frac{\mu_{\mathrm{eq}}}{k T}$ We now discuss these two regimes in detail.

3 2.1 Partial wetting $(D>\xi)$. - If the attraction between the monomers and the wall is not too strong, $D$ is larger than $\xi$, the derivative can be neglected in equation $\left(10^{\prime}\right)$ and the concentration inside the adsorbed globule is approximately constant The osmotic pressure being zero, this concentration is the same as that in a non adsorbed chain $\phi_{d}$, this imposes the value $\varepsilon=\frac{\mu_{\text {eq }}}{k T}$ As noted in the previous section, the free energy of the chain is dominated by surface terms The chains adopts a configuration that minimizes its total surface energy quite similarly to a droplet of an immiscible liquid It forms thus a spherical cap on the wall characterized by its contact 'angle $\theta$. As for simple liquids the contact angle is given by the classical Young law

$$
\gamma_{\mathrm{sw}}-\gamma_{\mathrm{pw}}=\gamma \cos \theta
$$

where $\gamma_{1}$ s the interfacial tension between the chain and the pure solvent given by equation'(5), $\gamma_{\mathrm{sw}}$ is the interfacial tension between the pure solvent and the wall and $\gamma_{\mathrm{pw}}$ the interfacial tension between the polymer chain and the wall. Notice that although we consider here a single chain, we describe it in terms of macroscopic concepts such as surface tensions and contact angles, this is however reasonable because we consider only length scales larger than the blob size $\xi$ which is the characteristic length scale of fluctuations, at these length scales both the interfaces between the chain and the solvent or the wall and the contact angle are well-defined quantities

The difference $\gamma_{\mathrm{sw}}-\gamma_{\mathrm{pw}}$ may be estimated from the thermal blob model. Each thermal blob of size $\xi$ in contact with the wall has $g \sim \xi^{2}$ monomers but only $g^{1 / 2}$ are in direct contact with the wall. If each of these monomers gains an adsorption energy $k T \delta \sim k T / D$ the total energy gained by a blob in contact with the surface is $g^{1 / 2} k T \delta \sim k T \xi / \check{D}$. This leads to a difference of interfacial tensions $\gamma_{\mathrm{sw}}-\gamma_{\mathrm{pw}} \sim k T / \xi D$ A more detailed calculation with a continuous layer of polymer similar to the one of section 3 below gives

$$
\gamma_{\mathrm{sw}}-\gamma_{\mathrm{pw}}=\frac{k T}{12 \xi D} \frac{a^{3}}{w} \text {. }
$$


The contact angle exists only if the spreading power $S=\gamma_{\mathrm{sw}}-\gamma_{\mathrm{pw}}-\gamma$ is negative [13] ; it thus exists when $D>\xi$ which corresponds to the partial wetting situation for usual wetting problems. Moreover this suggests a wetting transition when $D$ is of order $\xi$ to the flat pancake configuration that corresponds to the complete wetting situation in the equivalent problem of wettıng by a simple liquid

32.2 Complete wetting $(D<\xi)$, - In the reverse limit where $D$ is smaller than $\xi$ the interaction terms are negligible in equation $\left(10^{\prime}\right)$ and the polymer adsorbs as a flat pancake.of thickness $D$ quite similarly to a Gaussian chain In the thermal blob model this can be understood as a limit where each blob adsorbs individually. The chain may then be viewed as a two dimensional chain of Gaussian blobs of size $D$ This thin film configuration is reminiscent of the thin films formed by fluids completely wettıng a wall Notice however that the finite thickness of the adsorbed polymer is due to the elasticity of the chain and not to the disjoining pressure that characterizes the long range character of the molecular interaction between the liquid and the solid. This disjoining pressure has been neglected here, we briefly discuss its role for thick wetting film' below, in the case of Van der Waals interactions

We now discuss the configuration of the adsorbed chain in the direction parallel to the wall. Although in the bulk the polymer is in a poor solvent in the adsorbed state it can' be either in a poor solvent or in a good solvent. As a polymer chain is confined the $\theta$ point shifts to lower temperatures [14], it is not obtained when $v=0$ but for a negative value $v_{\theta}$ such that the correlation length $\xi$ is of order $D$

$$
v_{\theta} \sim-\frac{w^{2}}{D a^{2}}
$$

The lowering of the critical point as the space dimension is reduced is a general result in the theory of phase transitions [15], for the $\theta$ point which corresponds to a tricritical point in the magnetic analog to polymers [16], it may be understood by the following qualitative argument. The $\theta$ point is the point where the interaction free energy between two Gaussian blobs of size $D$ vanishes. This interaction may be estimated by integrating the interaction free energy equation $\left(4^{\prime}\right)$ inside a volume of size $D$ where the chain is Gaussian, $\frac{F_{\mathrm{b}}}{k T} \sim \frac{v D}{a^{4}}+\frac{w^{2}}{a^{6}}$, when $D$ is small it vanishes for a negative value of $v$ of order $v_{\theta}$ indicating the shift to lower temperature of the $\theta$ point, in the limit where $D$ is infinite (in the bulk) the $\theta$ point 1 s obtained when $v$ vanishes.

If the solvent is poor enough, so that $v$ is smaller than $v_{\theta}$, the two dimensional adsorbed chain is in a poor solvent and is a two-dimensional collapsed chain of blobs of size $D$ with a radius $R=N^{1 / 2} a$ If the excluded volume parameter, $v$, has an intermediate value $v_{\theta}<v<0$ the adsorbed polymer is in a good solvent and shows the two-dimensional excluded volume statistics with a radius $R \sim a N^{3 / 4}\left(\left(v-v_{\theta}\right) a^{-3}\right)^{1 / 4}(D / a)^{-1 / 4}$

One should note that the flat pancake configuration exists only in the limit $D<\xi$, the scaling arguments that.we have just given are thus not sufficient to decide whether one can reach the two-dimensional $\theta$ point in this limit and thus observe two-dimensional collapsed chains. A more refined theory including all numerical coefficients would be necessary, this is far beyond the scope of this work, we will assume in the following that both the good solvent and the poor solvent behaviors can exist 'on the surface.

\section{Adsorption of a polymer solution in a poor solvent.}

We now discuss the adsorption of a poor solvent polymer solution If the concentration is high enough the solution separates into two phases and can undergo a wetting transition very 
similar to that observed in simple liquid binary mixtures. Above the transition a wetting film of macroscopic thickness forms on the wall. As the concentration is decreased below that of the dilute coexisting phase, the thickness of the wetting film decreases and the film eventually becomes a dilute solution of adsorbed polymer chains.

4.1 WETTING TRANSITION OF A POLYMER SOLUTION - We consider a solution in a poor solvent on the dilute branch of the coexistence curve The chemical potential of the solution far from the wall has the coexistence value $\mu_{\text {eq }}$ and its osmotic pressure may be neglected. The concentration profile of the solution close to the wall is obtained by minimization of the grand canonical free energy per unit surface

$$
\Omega=\Omega_{\mathrm{S}}+\int \mathrm{d} z\left\{F-\mu_{\mathrm{eq}} \phi\right\}
$$

where $\Omega_{\mathrm{S}}$ is the direct surface contribution due to the attraction of the monomers, $F$ is the generalized Flory-Huggins free energy given by (4) and $\phi$ the local concentration. This is the generalization to polymers of the Cahn-Hilliard approach of simple liquids and has been already studied for polymers by several authors [17-22]. We only give here the essential results.

Minımızıng the free energy (14) and neglectıng the entropy term leads to

$$
\mu_{\mathrm{eq}} \psi=-\frac{a^{2}}{6} \frac{\partial^{2} \psi}{\partial z^{2}}+v \psi^{3}+\frac{w^{2}}{2} \psi^{5}
$$

where we again have introduced the order parameter $\psi=\phi^{1 / 2}$ This is the same as equation (10) used to study the adsorption of a single chain where $\varepsilon$ has been replaced by the chemical potential at coexistence $\mu_{\mathrm{eq}}$.

The boundary condition is obtained by minimizing $\Omega$ with respect to the surface concentration $\phi_{\mathrm{S}}$. Expanding $\Omega_{\mathrm{S}}$ in powers of concentration $\Omega_{\mathrm{S}}=\gamma_{0}-k T \delta \phi_{\mathrm{S}}$ we obtain a boundary condition similar to (9) where $D$ is related to $\delta$ by the same relation as above, $D=\frac{a^{2}}{6 \delta}$.

In the Cahn theory of the wetting transition [23] one obtains an equation governing the concentration $\phi$ very similar to (15) but the variable here is the order parameter $\psi$ and not the concentration, also the boundary condition is different, this is due to the different form of the concentration gradient terms in the free energy; these differences do not lead to a qualitatively different behavior.

In general equation (15) has three solutions. One corresponds to the dilute equilibrium phase in contact with the wall, one to a macroscopic film of the dense equilibrium phase in contact with the wall and the third one is always unstable.

At low temperature when $2\left(2^{1 / 2}+1\right) \xi<D$ the wetting film is unstable, the concentration decreases rapidly from the wall to the concentration of the dilute phase over a distance $\xi$ We already mentioned however that the dilute side of the coexistence curve is only poorly described by the Flory free energy.

The wetting transition occurs when $2\left(2^{1 / 2}+1\right) \xi=D$ or for a value of the excluded volume, $v_{\mathrm{w}}=-\frac{2\left(2^{1 / 2}+1\right)}{3} \frac{a w}{D}$, it is here first order. At a larger value of $\xi$ a film of the dense phase at the concentration $\phi_{\mathrm{d}}$ forms on the wall. The concentration on the surface $\phi_{\mathrm{S}}$ is given by

$$
\phi_{S}=\frac{-3 v}{2 w^{2}}+\frac{a w}{D}
$$


The concentration decreases smoothly over a distance $D$ to the equilibrium value $\phi_{\mathrm{d}}$. Notice that the second term in $\phi_{\mathrm{S}}$ dominates and is equal to the concentration in a Gaussian blob of size $D$ Qualitatıvely one can describe the wetting film as one layer of gaussian blobs of size $D$ on the wall and then a uniform closepacked solution of blobs of size $\xi$.

This shows that as in the bulk, the behavior of an isolated chain at an interface is very similar to that of the dense equilibrium phase, in both cases, we expect a wetting transition when $D \sim \xi$.

4.2 GROWTH OF THE WETTING LAYER, PREWETTING TRANSITION - We start from an extremely dilute solution in a poor solvent where the chains adsorb individually and increase the concentration. We consider only the complete wetting case $0>v>v_{\mathrm{w}}$ where the chains form flat pancakes and as announced earlier we suppose that the $\theta$ point in two dimensions is such that $v_{\theta}>v_{\mathrm{w}}$. As the bulk concentration is increased the adsorbed layer is first a two dimensional dilute solution then a semi-dilute polymer solution either in a good solvent $\left(v>v_{\theta}\right)$ or in a poor solvent $\left(v<v_{\theta}\right)$. By further increase of the bulk concentration, it forms a wettıng layer of the dense equilibrium phase with a finite thickness that diverges when the coexistence curve is reached.

42.1 Dilute adsorbed layer in a good solvent - If $v>v_{\theta}$ the adsorbed chains form a two dimensional solution in a good solvent, we call $\sigma$ the number of monomers per unit area. For sake of simplicity we discuss only the strong excluded volume limit $-v \ll-v_{\theta}$ which 1 s rather similar to what is obtained by adsorption from a good solvent as discussed by Bouchaud and Daoud [24].

The radius of an isolated pancake follows the two dimensional excluded volume statistics

$$
R \sim N^{3 / 4} D^{-1 / 2}
$$

The chemical potential of one adsorbed chain is

$$
\frac{\mu_{\mathrm{ch}}}{k T}=-N \frac{a^{2}}{6 D^{2}}+\log \frac{\sigma a^{2}}{N}-N \frac{\mu_{\text {eq }}}{k T}
$$

where the first term accounts for the wall attraction and the second one for the entropy of mixing and the zero of chemical potential has been taken at the coexistence value

The value of the surface concentration is obtained by equating the chemical potential to that of the bulk given by equation (7) The surface concentration increases then linearly with the bulk concentration $\phi$

$$
\sigma \sim \phi \exp N \frac{a^{2}}{6 D^{2}}
$$

When the surface concentration reaches the overlap concentration $\sigma^{*} \sim N / R^{2} \sim N^{-1 / 2} D$, the adsorbed layer becomes a semidilute solution. The standard scaling laws for semidilute solutions give the correlation length and the free energy per unit area

$$
\xi_{\mathrm{S}}=\left(\sigma a^{2}\right)^{-3 / 2} D, \quad F=\frac{k T}{\xi_{\mathrm{S}}^{2}}
$$

The total chemical potential of one chain is then

$$
\mu_{\mathrm{ch}}=-N \frac{a^{2}}{6 D^{2}}+N\left(\sigma a^{2}\right)^{2}\left(\frac{a}{D}\right)^{2}-N \frac{\mu_{\mathrm{eq}}}{k T}
$$


and the surface concentration increases logarithmically with the bulk concentration as $\sigma=\sigma^{*} \cdot\left[N \frac{{ }^{\prime} a^{2}}{6 D^{2}}+\log \phi\right]^{1 / 2}$ untıl it reaches a contınuous layer of blobs of size $D$ when $\sigma a^{2} \sim 1$.

4.2.2 Dilute adsorbed layer in a poor solvent, prewetting transition. - When $v<v_{\theta}$ the two dimensional polymer solution is in a poor solvent and as in a three dimensional poor solvent, it has a tendency to phase separate between a dense polymer phase and a dilute polymer phase. We do not wish to discuss here the behavior in the vicinity of the $\theta$ point in details but we want to give a qualitative description of the phase separation In the language used for wetting phenomena with simple fluids binary mixtures, this transition is the so-called prewetting transition [8].

In the limit of very high molecular weight the critical point tends to the $\theta$ point $v_{\theta}$ and the critical concentration is the overlap concentration of the chains (with the two dimensional $\theta$ statistics) The universality class is the Isıng universality class in two dimensions as for usual demixing. transitions.

We focus now on the very poor solvent limit $\left(v-v_{\theta}\right) \sim v_{\theta}$ where an analysis very similar to that proposed above in 3 dimensions can be made. In this limit, the dense phase is a closepacked assembly of blobs of size $D\left(\sigma a^{2} \sim 1\right)$ The chemical potential in the dense phase 1s of order $k T$ per blob or per monomer $\frac{\mu}{k T} \sim \frac{a^{2}}{D^{2}}$

The dilute phase is a very dilute solution of collapsed chains with a radius $R \sim N^{1 / 2} a$. If we take as a reference state the chemical potential of the dense phase, the free energy of a chain is dominated by the two dimensional interfacial free energy that we describe in terms of a line tension $\tau \sim \frac{k T}{D}$, the chemical potential of the dilute phase is

$$
\frac{\mu_{\mathrm{ch}}}{k T}=\log \frac{\sigma a^{2}}{N}+\tau 2 \pi R
$$

This gives a surface concentration decreasing-exponentially with the molecular weight as $\sigma \sim \exp -N^{1 / 2} a / D$. When this concentration is reached, the surface concentration jumps discontinuously to that of a dense layer of blobs.

The surface prewetting transition occurs when the chemical potential of a chain in the bulk is of order $\frac{\mu_{\mathrm{cb}}}{k T} \sim-N \frac{a^{2}}{D^{2}}$ 1.e. , for a concentration in the bulk of order $\dot{\phi} \sim \exp \left[-\alpha N \frac{a^{2}-}{D^{2}}\right]$ where $\alpha$ is an unknown numerical constant

4.2.3 Polymer wetting film. - In both cases where the adsorbed polymer layer is in a good or a poor solvent, the surface layer becomes a closepackıng of Gaussian blobs of size $D$ when the bulk chemical potential is of order $-N \frac{a^{2}}{D^{2}}$ If the bulk concentration is further increased, the polymer film on the surface grows in thickness and a wetting film of the dense coexistence phase forms on the wall. In order to study the properties of this prewetting film, we use the same method as that used to study the wetting transition at coexistence We minimize the grand canonical free energy per unit surface

$$
\Omega=\Omega_{\mathrm{S}}+\int \mathrm{d} z\left\{F-\left(\mu+\mu_{\mathrm{ex}}\right) \phi\right\} .
$$

The only difference with equation (14) is that the chemical potential does not have the 
coexistence value $\mu_{\text {eq }}$ but a lower value, the bulk concentration being smaller than that of the dilute branch of the phase diagram ( $\mu=\mu_{\text {ch }} / N$ where $\mu_{\text {ch }}$ is given by (7))

The minimization of equation (14') with the boundary condition given by (9) gives the following characteristics for the prewetting film

Close to the surface in a layer of size $D$ the concentration is of the order of that in a Gaussian blob of size $D$ as given by (16) The concentration is then approximately constant and equal to that of the dense phase $\phi_{d}$ over a thickness $L$. It then relaxes exponentially to the bulk concentration over a correlation length $\xi$

We have calculated the surface concentration $\sigma$ by direct minımization of $\left(14^{\prime}\right)$ we find

$$
\sigma=\frac{a}{2 w} \log \left\{\frac{\left(1 / D^{2}-1 / l^{2}\right)^{1 / 2}+1 / D}{-1 / 2 \xi+\left(1 / 4 \xi^{2}+1 / l^{2}\right)^{1 / 2}}\right\}
$$

where for convenience the chemical potential $\mu$ is expressed as a length $l=a /(-6 \mu)^{1 / 2}$

When $l$ is of order $D$ (or $\mu$ of order $-\frac{a^{2}}{D^{2}}$ ), the surface concentration reaches that of a continuous layer of Gaussian blobs of size $D, \sigma \sim 1 / a^{2}$ (with a logarithmic correction here).

In the range $D<l<\xi$ the surface concentration increases smoothly as $\sigma \sim \log l / D$, the thickness of the layer is proportional to $l \sim a N^{1 / 2}(-\log \phi)^{-1 / 2}$; it thus crosses over smoothly to the behavior discussed above in sections 421 or 4.2.2.

When the bulk concentration is close to the dilute branch of the coexistence curve $\phi_{\text {eq }}(1>\xi)$, the thickness of the layer can be defined as $L=\frac{\sigma}{\phi_{\mathrm{d}}}$. The surface concentration and thus the thickness of the wetting layer diverge when $\phi$ reaches $\phi_{\text {eq }}$ as

$$
\sigma \sim L / \xi \sim \log \left(\mu_{\mathrm{eq}}-\mu\right) \sim \log \left[\left(\phi_{\mathrm{eq}}-\phi\right) / \phi_{\mathrm{eq}}\right] .
$$

This logarithmic divergence is classical for simple flu1d mixtures when the interactions are short range It is however not often observed because of the long range character of the interaction between the monomers and the wall [25]. We consider only briefly the case of Van der Waals forces.

We assume that a prewetting film of thickness $L$ forms on the solid and that the long range character of the Van der Waals forces give only a small perturbation to the concentration profile calculated above [26]. We write 1 ts free energy per unit surface as a function of $L$

$$
\frac{F}{k T}=\phi_{\mathrm{d}}\left(\mu-\mu_{\mathrm{eq}}\right) L+P(L) .
$$

The first term is due to the chemical potential difference with the coexistence. When $L \gg \xi$ we can neglect the effect of the finite thickness $L$ that gives a correction decaying as $\exp -L / \xi$. The second term is the Van der Waals interaction which varies with thickness as

$$
P(L)=\frac{A^{\prime}}{12 \pi L^{2}} \text {. }
$$

$A^{\prime}$ is the relevant Hamaker constant that is in general positive in conditions of complete wetting. In a first approximation, if the solid is much more polarizable than both the polymer and the solvent $A^{\prime}$ can be related to the Hamaker constant $A$ of a molten polymer layer by $A^{\prime}=\phi_{\mathrm{d}} a^{3} A$

The equilibrium thickness is obtained by minimization of (25), as in simple liquid mixtures, the thickness of the film diverges at coexistence as $L=a\left[\frac{A}{6 \pi\left(\mu-\mu_{\mathrm{eq}}\right)}\right]^{1 / 3}$. 


\section{Concluding remarks and application to block copolymers.}

5.1 SUMMARY OF THE RESULTS. - The most important result that we have obtained is that the adsorption of a polymer chain in a poor solvent can be viewed as a wetting problem. The wetting behavior is governed by the ratio of the two length scales of the problem, the adsorption length $D$ and the size of the thermal blob $\xi$

When $D$ is larger than $\xi$, the polymer chain behaves exactly as a small liquid drop of thermal blobs that wets partially the solid surface All the properties of the polymer chain including the behavior at an interface are obtained quantitatively by considering one chain as a small drop of the equilibrium phase on the concentrated branch of the phase diagram

When $D$ is smaller than $\xi$, which is the analog of complete wetting, the chain adopts a flat configuration on the interface The configuration of the chain parallel to the surface is that of a two dimensional chain either in a good solvent if $v>v_{\theta}$ and in a poor solvent if $v<v_{\theta}$. Although the configuration is similar to that observed with a completely wetting liquid, note that the various interfacial tensions are not well defined in this case, the analogy with wetting is weaker.

Our theory has been limited to scaling laws and we have not been able to discuss the precise nature of the wettıng transition for a single chain and to decide whether the two dimensional poor solvent behavior $\left(v<v_{\theta}\right)$ always exists. A more detailed study of equation $\left(10^{\prime}\right)$ would certainly be needed to study the nature of the wetting transition

We also have studied the concentration effects and the formation of a continuous wetting layer on the wall We have predicted a prewetting transition at the $\bar{\theta}$ point of the adsorbed layer $v_{\theta} \sim-a / D$ The wetting behavior of a polymer solution has been calculated in details using Flory-Huggins mean field theory, the wettıng transition has been shown to be first order It is qualitatively very similar to the wetting behavior of binary mixtures of simple liquids.

One must note that there is little hope to observe these effects directly on a polymer solution in a poor solvent At a given temperature below the $\theta$ point, the bulk concentration at which phase separation occurs is so low that for long polymers it is not experimentally accessible

However some of the effects described here could be easier to observe by studying the adsorption of diblock copolymers in a selective solvent where the adsorbing block is in a-poor solvent that we briefly discuss below The results on single chain adsorption could also be compared to numencal simulations currently in progress [27].

5.2 BLOCK COPOLYMER ADSORPTION IN A SELECTIVE SOLVENT. - We consider the copolymers that we have studied in the highly selective solvent limit in references [1] and [2]. The soluble block B is repelled by the solid surface it has $N_{\mathrm{B}}$ monomers and its natural radius is the Flory radius, $R_{\mathrm{B}} \sim N_{\mathrm{B}}^{3 / 5}$. The other block A containing $N_{\mathrm{A}}$ monomers is in a poor solvent and is attracted by the wall

In order to describe the bulk properties, one can use the thermal blob model and generalize easily the results of reference [1] The copolymer forms micelles above a critical micellar concentration $\phi_{\mathrm{cmc}} \sim \exp -\left(N_{\mathrm{A}} a^{2} / \xi^{2}\right)^{2 / 3}$, the chemical potential of one chain in the micellar phase is $\mu_{\mathrm{cmc}} \sim k T\left(N_{\mathrm{A}} a^{2} / \xi^{2}\right)^{2 / 5}$ where we have chosen the origin of free energy for the $\mathrm{A}$ chains at the coexistence value of the chemical potential $N \mu_{\text {eq }}$ The c.m.c. is small only if $\xi<N_{\mathrm{A}}^{1 / 2} a$, micelle formation in dilute solution occurs only if the temperature is lower than the consolute point of the equivalent $\mathbf{A}$ homopolymer in the same solvent. At a higher temperature, mesophase formation occurs in a semidilute solution 
In contact with a wall, a micellar solution forms an adsorbed film with a double layer structure, an A layer in direct contact with the wall and a B brush dangling into the solvent The existence of the B blocks acts as an external field that decreases the thickness of the wetting layer of the A block; the stretching energy of the B block given in the Alexander blob model by $\mu_{\mathrm{S}} \sim k T N_{\mathrm{B}}\left(\frac{\sigma a^{2}}{N_{\mathrm{A}}}\right)^{5 / 6}$ must be added to the chain chemical potential when the $\mathrm{B}$ chains come into contact $\left(\sigma>N_{\mathrm{A}} / N_{B}^{-6 / 5}\right)$.

If $N_{\mathrm{B}}<\frac{N_{\mathrm{A}}^{11 / 6} a^{2}}{D^{2}}$ the adsorbed A layer is continuous, one can then combine the results of the highly selective solvent theory to the thermal blob model to describe the geometrical structure of the surface copolymer layer.

If $N_{\mathrm{B}}>\frac{N_{\mathrm{A}}^{11 / 6} a^{2}}{D^{2}}$ the adsorbed A layer in equilibrium with a micellar solution is discontinuous; one can thus form a dilute solution of adsorbed $\mathrm{A}$ chains in a poor solvent. The surface concentration is obtained by equating the chemical potentials in the bulk and on the surface. Using equation (18) we obtain

or

$$
\frac{\mu_{\mathrm{cmc}}}{k T}=-N_{\mathrm{A}} \frac{a^{2}}{6 D^{2}}+N_{\mathrm{B}}\left(\frac{\sigma a^{2}}{N_{\mathrm{A}}}\right)^{5 / 6}
$$

$$
\sigma=\frac{N_{\mathrm{A}}^{11 / 5}}{N_{\mathrm{B}}^{6 / 5}}\left(\frac{a}{D}\right)^{12 / 5}
$$

The prewetting transition should also exist with copolymers when $N_{\mathrm{B}}<N_{A}^{4 / 3}\left(\frac{a}{D}\right)$ and $v<v_{\theta}$ When increasing the bulk concentration from zero the surface concentration $\sigma$ shows a first order transition between a very low value and a continuous layer of thickness $D$. The bulk concentration where this transition occurs, although larger than for homopolymers in a poor solvent, remains much lower than the c.m.c. and might be difficult to observe experimentally.

\section{References}

[1] Marques C, Joanny J. F, Leibler L, Macromolecules 21 (1988) 1051

[2] JOHNER A, JoANNY J F, Macromolecules, in press

[3] See for instance PARSONNAGe E et al., preprint (1990)

[4] Flory P J, Principles of polymer Chemistry (Cornell University press, Ithaca) 1971.

[5] De Gennes P. G., Scaling Concepts in Polymer Physics (Cornell University press, Ithaca) 1985

[6] Sun S. et al, $J$ Chem Phys 73 (1980) 5971

[7] Lifschitz I M, Grosberg A, Khokhlov A, Rev. Modern Phys 50 (1978) 683

[8] See for example the reviews by M SCHICK and D BEYSENS, in "Liquids at interfaces " (Les Houches summer school, 1988) J. Charvolın, J F Joanny Eds (North Holland) in press

[9] JoAnNy J F., Thèse de $3^{\circ}$ cycle Unıversité Pans VI (1978).

[10] MOORE M, J Phys A 10 (1977) 305

[11] Doi M , EDwards S., The theory of polymer dynamics (Oxford University press, Oxford) 1986

[12] De Gennes P. G, Rep. Prog. Phys 32 (1969) 187

[13] Rowlinson J., Widom B, Molecular Theory of Capıllanty (Oxford Unıversity press, Oxford) 1982 
[14] The lowerng of the $\theta$ point has already been noted in framework of polymer adsorption by VAN DIEREN, F , KREMER K , Europhys Lett 4 (1987) 569

[15] Toulouse, $G_{1}$, PFeuty $P$, Introduction au groupe de renormalisațion et à ses applications (Presses $\therefore$ Universitaires, de Grenoble, Grenoble) 1975

[16] DES Cloizeaux J., JANINK G, Les polymères en solution · leur modélisation et leur structure (Editions de Physique, Orsay) 1987,

[17] NakANishi H, Pincus P J, Chem Phys 79 (1983) 997

[18] SCHMITT I, Binder K, J Phys France 46 (1985) 1631

[19] KLeIN J , PINCuS P, Macromolecules 15 (1982) 1129

[20] Ingersent K, Klein J , Pincus P, Macromolecules 19 (1986) 1374

[21] Carmesin I, Noolandi I, Macromolecules 22 (1989) 1683

[22] Cohen S, Muthukumar M, $J$ Chem. Phys 90 (1989) 5749

[23] CAHN J, J Chem Phys 66 (1977) 3667

[24] Bouchaud E, Daoud M, J. Phys France 48 (1987) 1991

[25] De Gennes P G, $J$ Phys Lett 42 (1981) L-337.

[26] DE Gennes P G, Macromolecules 14 (1981) 1637

[27] Muthukumar M , Private communication 